\title{
XVII. Akademische Tagung deutschsprechender Hochschullehrer in der Gynäkologie und Geburtshilfe
}

\author{
Salzburg, 3.-5. Oktober 2001 \\ and Obstetrics, Salzburg, October 3-5, 2001

\section{P. Husslein} \\ Universitätsklinik für Frauenheilkunde, Wien, Österreich
}

XVIth Academic Meeting of German-Speaking University Teachers of Gynaecology

Die Organisation der XVII. Akademischen Tagung Deutschsprechender Hochschullehrer war und ist für mich eine ausserordentlich grosse Ehre, und ich möchte die Gelegenheit wahrnehmen, mich beim deutschen Ordinarienkonvent für dieses grosse Vertrauen zu bedanken. Ich freue mich auch, dass nach einigem Zögern beschlossen wurde, bis auf weiteres die Tagung abwechselnd in der BRD, der Schweiz und eben in Österreich durchzuführen. Dies ist in Zeiten des Zusammenschlusses Europas ein wichtiges - gutes - Zeichen für die kleinen Länder.

Eine so renommierte Tagung und die Zäsur der Jahrtausendwende sind ein guter Anlass, um ein paar Überlegungen zur Entwicklung der Frauenheilkunde anzustellen. Aus der Fülle möglicher Themen möchte ich drei besonders hervorheben:

- Eine der grössten Herausforderungen zum heutigen Zeitpunkt ist es, die notwendige Subspezialisierung unseres Faches zuzulassen, ja sogar zu fördern, ohne gleichzeitig die Subdisziplinen allzu stark auseinanderdriften zu lassen. Gerade in der Forschung, aber auch im Bereich der Patientenbetreuung - insbesondere auf dem Niveau eines Zentralkrankenhauses - haben Ausmass und Tiefe unseres Wissens heute derart zugenommen, dass ein Überblicken des Gesamtfaches für einen Einzelnen, aber eben auch für eine ganze Abteilung auf einem für Spitzenleistungen notwendigen Niveau nicht mehr möglich ist. Daraus leitet sich die unabdingbare Notwendigkeit einer

\begin{tabular}{ll}
\hline KARGER & $($ 2001 S. Karger AG, Basel \\
Fax +4161306 1234 & \\
$\begin{array}{l}\text { E-Mail karger@karger.ch } \\
\text { www.karger.com }\end{array}$ & $\begin{array}{l}\text { Accessible online at: } \\
\text { www.karger.com/journals/ggr }\end{array}$
\end{tabular}

Spezialisierung und sogar immer mehr einer Subspezialisierung ab. Verbunden damit steigt aber gleichzeitig für den einzelnen Wissenschaftler und Arzt die Gefahr, zwar immer mehr Details seines eigenen Spezialgebietes zu kennen, dafür aber den Überblick über das Gesamtfach und die notwendigen Vernetzungen im Gesamtsystem zu verlieren.

Patentlösungen zur Überwindung der durch eine Spezialisierung auftretenden Barrieren und Kluften zwischen den einzelnen Disziplinen gibt es naturgemäss keine. Es wird von der Institution, in der man arbeitet, von der dort vorhandenen Organisationsstruktur, vor allem aber von den handelnden Personen abhängen, ob die grosse Herausforderung, eine Spezialisierung zuzulassen, aber den Anspruch der Einheit des Faches aufrecht zu erhalten, gelingen wird. Abschreckende Beispiele anderer Fächer sollten uns zu denken geben.

- Der zukünftige Stellenwert der Forschung in den klinischen Fächern wird unter anderem auch davon abhängen, ob es gelingt, die Grundlagenforschung an den jeweiligen Kliniken zu fördern und Bindeglieder zwischen Klinik und Labor herzustellen bzw. dort, wo solche Brücken vorhanden sind, diese zu festigen und weiter zu entwickeln.

Auch hier besteht die Gefahr, dass die Grundlagenforschung sich von der Klinik und von den dort relevanten Fragestellungen in ihrer Liebe und Zuwendung zum Detail immer weiter entfernt und umgekehrt, dass der Kliniker durch seine zumeist geringe Beherrschung der Metho-

o. Univ. Prof. Dr. Peter Husslein, Vorstand der Universitätsklinik für Frauenheilkunde Wien, Währinger Gürtel 18-20 A-1090 Wien (Österreich), Tel. +43 1404002821

Fax+43140400 2862, E-Mail peter.husslein@akh-wien.ac.at 
den der Grundlagenforschung immer mehr die Fähigkeit verliert, Ergebnisse aus dem Labor nachzuvollziehen, zu bewerten, aber unter Umständen auch kritisch zu hinterfragen.

Dabei hat es sich bewährt, beispielsweise die Forschungsflächen in räumliche Nähe zum Kreisssaal, Operationssaal oder zu den Ambulanzen bzw. sonstigen Bereichen der Klinik einzurichten, weil sonst die räumliche Distanz oft auch eine emotionelle und inhaltliche Distanz nach sich zieht. Auch eine Freistellung einzelner Mitarbeiter für ein halbes oder ganzes Jahr für die Grundlagenforschung ist für eine holistische Betrachtung des Faches von unschätzbarem Wert. Allzu klinisch ausgerichtete Abteilungsleiter oder Klinikchefs sollten hier ermuntert werden, den dadurch bedingten Personalausfall im Patientenbetreuungsbereich unter dem Blickwinkel der umfassenden Aus- und Weiterbildung der jeweiligen Mitarbeiter zu sehen.

- Eine besondere Herausforderung für unser Fach besteht meiner Ansicht nach auch darin, die diskrepante Entwicklung der Frauenheilkunde, insbesondere der Geburtshilfe in den Ländern der Ersten und Dritten Welt, zunächst ernsthaft zur Kenntnis zu nehmen und im Weiteren die geradezu erschütternden Niveauunterschiede in der Versorgung der Bevölkerung ein wenig ausgleichen zu helfen.

Während wir in Europa und Nordamerika zum Beispiel darüber diskutieren, ob aus Annehmlichkeitsgründen oder zur Schonung des Beckenbodens ein Kaiserschnitt durchgeführt werden kann oder soll, versterben Mütter in der Dritten Welt heute noch immer auf dem Weg von ihrem Geburtsort in eine Versorgungseinrich- tung an postpartalen Blutungen. Einer ganz rezenten $\mathrm{Zu}-$ sammenstellung der Weltgesundheitsorganisation über die mütterliche Mortalität in den verschiedenen Ländern entnimmt man, dass z.B. 1995 weltweit pro Jahr eine halbe Million Frauen verstorben sind, wobei in den industrialisierten Ländern die mütterliche Mortalitätsrate pro 100000 Lebendgeburten bei 12 (und wahrscheinlich darunter) lag, während sie in Afrika oder ähnlich schlecht entwickelten Ländern auf bis zu 1200 und darüber geschätzt wird.

Während in den Ländern der Ersten Welt ein immer grösserer Ressourceneinsatz notwendig ist, um immer geringeren Nutzen zu stiften, befindet man sich auf der Kosten-/Nutzen-Kurve in den Ländern der Dritten Welt noch in einem sehr steilen Bereich: Einfache Massnahmen können dort erfreulicherweise noch erhebliche Verbesserungen für die betroffene Bevölkerung nach sich ziehen. Wir sollten dies bei allem Verständnis für die Notwendigkeit laufender Verbesserungen für die Gesundheit der sich uns anvertrauenden Frauen nie vergessen.

Die Hochschullehrertagung hat traditionell die Aufgabe, den Gedankenaustausch zwischen den Universitäten, aber auch zwischen jüngeren und arrivierteren Universitätslehrern zu ermöglichen.

Ich hoffe, dass mit der Auswahl insbesondere der mündlichen Beiträge der vorliegenden Tagung in Salzburg auch ein kleines Mosaiksteinchen zum Zusammenhalten unseres Faches gesetzt werden konnte und dass es auch gelingt, die Motivation der Teilnehmer zu erhöhen, sich ein bisschen über das eigene Spezialgebiet hinauszuwagen. 